\title{
Strichartz and smoothing estimates for Schrödinger operators with large magnetic potentials in $\mathbb{R}^{3}$
}

Received November 29, 2006 and in revised form February 14, 2007

Abstract. We present a novel approach for bounding the resolvent of

$$
H=-\Delta+i(A \cdot \nabla+\nabla \cdot A)+V=:-\Delta+L
$$

for large energies. It is shown here that there exist a large integer $m$ and a large number $\lambda_{0}$ so that relative to the usual weighted $L^{2}$-norm,

$$
\left\|\left(L(-\Delta+(\lambda+i 0))^{-1}\right)^{m}\right\|<1 / 2
$$

for all $\lambda>\lambda_{0}$. This requires suitable decay and smoothness conditions on $A, V$. The estimate (2) is trivial when $A=0$, but difficult for large $A$ since the gradient term exactly cancels the natural decay of the free resolvent. To obtain (2), we introduce a conical decomposition of the resolvent and then sum over all possible combinations of cones. Chains of cones that all point in the same direction lead to a Volterra-type gain of the form $\left(m !^{-\varepsilon}\right.$ with $\varepsilon>0$ fixed. On the other hand, cones that are not aligned contribute little due to the assumed decay of $\widehat{A}$. We make no use of micro-local analysis, but instead rely on classical phase space techniques. As a corollary of (2), we show that the time evolution of the operator in $\mathbb{R}^{3}$ satisfies global Strichartz and smoothing estimates without any smallness assumptions. We require that zero energy is neither an eigenvalue nor a resonance.

\section{Introduction}

Magnetic Schrödinger operators on $L^{2}\left(\mathbb{R}^{d}\right)$ are of the form

$$
H=-\Delta+i(A \cdot \nabla+\nabla \cdot A)+V=-\Delta+L .
$$

They model nonrelativistic magnetic effects in quantum mechanics and have been much studied in the physics literature. The seminal paper [2] discusses the case of constant magnetic fields (see also [6]). The scattering theory for decaying magnetic fields is discussed

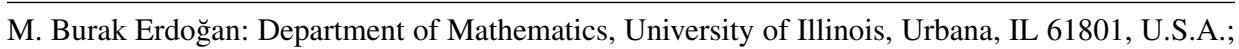
e-mail: berdogan@math.uiuc.edu

M. Goldberg: Department of Mathematics, Johns Hopkins University, Baltimore, MD 21218, U.S.A.; e-mail: mikeg@math.jhu.edu

W. Schlag: Department of Mathematics, University of Chicago, 5734 South University Avenue, Chicago, IL 60637, U.S.A.; e-mail: schlag@math.uchicago.edu

Mathematics Subject Classification (2000): 14 J29 
in [19]. More recent results on scattering by magnetic potentials are [24] and [30]. The review [7] contains a long list of references.

There has been much activity surrounding dispersive estimates for the case $A=0$ under suitable decay (and also regularity when $d \geq 4$ ) assumptions on $V$. In fact, in that case the harder $L^{1}\left(\mathbb{R}^{d}\right) \rightarrow L^{\infty}\left(\mathbb{R}^{d}\right)$ estimate is now known in all dimensions $d \geq 1$ under the condition that zero energy is neither an eigenvalue nor a resonance (and there are now also results in the case when the latter assumption does not hold). The seminal paper for this class of estimates is [14] and we refer the reader to [25] for a survey of more recent work.

On the other hand, much less is known when $A \not \equiv 0$. In [27] and [9] Strichartz and smoothing estimates were obtained for small $A$ and $V$. In this paper we prove the following theorem:

Theorem 1. Let $A$ and $V$ be real-valued such that for all $x, \xi \in \mathbb{R}^{3}$,

$$
\begin{aligned}
\langle x\rangle|A(x)|+|D A(x)|+|V(x)| & \lesssim\langle x\rangle^{-8-\varepsilon}, \\
\sum_{|\alpha| \leq 2}\left|D^{\alpha} \widehat{A}(\xi)\right| & \lesssim\langle\xi\rangle^{-3-\varepsilon},
\end{aligned}
$$

for some $\varepsilon>0$. Furthermore, assume that zero energy is neither an eigenvalue nor a resonance of $H$. Then, with $P_{c}$ being the projection onto the continuous spectrum,

$$
\left\|e^{i t H} P_{c} f\right\|_{L_{t}^{q}\left(L_{x}^{p}\right)} \lesssim\|f\|_{L^{2}\left(\mathbb{R}^{3}\right)}
$$

provided $(p, q)$ are admissible, i.e., $2 / q+3 / p=3 / 2$ and $2 \leq p<6$. Moreover, the inhomogeneous Strichartz estimates

$$
\left\|\int_{-\infty}^{t} e^{i(t-s) H} P_{c} F(s) d s\right\|_{L_{t}^{q}\left(L_{x}^{p}\right)} \lesssim\|F\|_{L_{t}^{\widetilde{q}^{\prime}}\left(L_{x}^{\tilde{p}^{\prime}}\right)}
$$

hold, where $(p, q)$ and $(\tilde{p}, \widetilde{q})$ are admissible as above. Finally, the Kato smoothing estimate

$$
\int_{0}^{\infty}\left\|\langle x\rangle^{-\sigma}\langle\nabla\rangle^{1 / 2} e^{i t H} P_{c} f\right\|_{2}^{2} d t \leq C\|f\|_{2}^{2}
$$

holds with $\sigma>4$.

It is well-known that Strichartz estimates are basic to the scattering theory of nonlinear equations. In this case, an immediate application would be to the nonlinear Schrödinger equation with a magnetic potential which has neither eigenvalues nor a zero energy resonance. See [13] for such an application when $A=0$ but $V$ is present.

The definition of zero energy being neither an eigenvalue nor a resonance is the usual one: there does not exist $f \in \bigcap_{\tau>1 / 2} L^{2,-\tau}\left(\mathbb{R}^{3}\right), f \not \equiv 0$, such that $H f=0$. In a sequel to this paper the authors will weaken the conditions on $A$ and $V$-in fact, for the sake of simplicity we have chosen to impose somewhat stronger conditions on $A$ and $V$ than the methods of this paper actually require. 
In the free case, Strichartz inequalities are typically proven by interpolating between the $L^{2}$-mass conservation law and an $L^{1} \rightarrow L^{\infty}$ dispersive estimate. Dispersive bounds are currently unknown for any $A \neq 0$, so a different approach is required here. We adopt an argument introduced in [23], where the validity of Strichartz inequalities is instead derived from Kato's theory of smooth perturbations.

The approach in this work is perturbative around the free case despite the fact that we make no smallness assumption. Fredholm theory provides some of the necessary analytical tools, and in other cases one is required to show that the perturbation series has an appropriately large radius of convergence. The main novel ingredient in this paper is a limiting absorption estimate for large energies. It is possible, for example, to argue that the perturbative effect of a scalar potential $V$ is small at high energies because it can be expressed in terms of an oscillatory integral. The same methods do not apply to the firstorder perturbation $L$ because the presence of an additional gradient negates every benefit arising from oscillation.

More precisely, recall that in [1] and [11] it is proved that for $H$ as in (3) under suitable decay conditions on $A$ and $V$ and with $\tau>1 / 2$,

$$
\sup _{\lambda \in\left[\delta, \delta^{-1}\right]}\left\|\langle\nabla\rangle\langle x\rangle^{-\tau}\left(H-\left(\lambda^{2}+i 0\right)\right)^{-1}\langle x\rangle^{-\tau}\langle\nabla\rangle\right\|_{2 \rightarrow 2} \leq C(\delta)<\infty
$$

provided there are no imbedded eigenvalues in the continuous spectrum. However, this is known due to recent work by Koch and Tataru [18]. It is well-known that this limiting absorption principle is of fundamental importance for proving dispersive estimates, at least for the case of large potentials. However, one needs to consider all real $\lambda$ instead of restricting to a compact interval in the positive halfline. To extend (9) toward zero energies is similar to the case $A=0$. This step requires the assumption on zero energy.

Note that (9) as stated cannot be extended to a semi-infinite interval since it would fail even for the free resolvent. Indeed, with $\tau>1 / 2$,

$$
\left\|\langle\nabla\rangle^{\alpha}\langle x\rangle^{-\tau}\left(H_{0}-\left(\lambda^{2}+i 0\right)\right)^{-1}\langle x\rangle^{-\tau}\langle\nabla\rangle^{\alpha}\right\|_{2 \rightarrow 2} \sim \lambda^{2 \alpha-1}
$$

for any $\alpha \in[0,1]$ and all $\lambda>1$. This shows that no more than one derivative in total can be gained here while still preserving a uniform upper bound. Furthermore, in the borderline case $\alpha=1 / 2$ there is no decay of the operator norm in the limit $\lambda \rightarrow \infty$.

We will adopt the shorthand notation

$$
R_{0}(z):=\left(H_{0}-z\right)^{-1}
$$

for the resolvent of the Laplacian. The resolvent of a general operator $H$ will be indicated by $R_{H}(z)$, or else $R_{L}(z)$ in the case where $H$ is specifically of the form $H_{0}+L$. Formally, the relationship between $R_{L}$ and $R_{0}$ is captured in the identity

$$
R_{L}(z)=\left(I+R_{0}(z) L\right)^{-1} R_{0}(z) .
$$

In this paper we extend (10) to $H=H_{0}+L$ for the class of first-order perturbations described in Theorem 11. A unified statement of the mapping properties of the resolvent of $H$ over the entire spectrum $\lambda>0$ is as follows. 
Theorem 2. Suppose $H$ is a magnetic Schrödinger operator whose potentials satisfy the conditions (4), (5). Then for $\tau>4$ and $\alpha \in[0,1]$,

$$
\sup _{\lambda>1} \lambda^{1-2 \alpha}\left\|\langle\nabla\rangle^{\alpha}\langle x\rangle^{-\tau}\left(H-\left(\lambda^{2}+i 0\right)\right)^{-1}\langle x\rangle^{-\tau}\langle\nabla\rangle^{\alpha}\right\|_{2 \rightarrow 2} \lesssim 1 .
$$

If one further assumes that zero is not an eigenvalue or resonance of $H$, then this bound can be extended to

$$
\sup _{\lambda \geq 0}\langle\lambda\rangle^{1-2 \alpha}\left\|\langle\nabla\rangle^{\alpha}\langle x\rangle^{-\tau}\left(H-\left(\lambda^{2}+i 0\right)\right)^{-1}\langle x\rangle^{-\tau}\langle\nabla\rangle^{\alpha}\right\|_{2 \rightarrow 2} \lesssim 1 .
$$

As a consequence, the spectrum of $H$ is purely absolutely continuous over the entire interval $[0, \infty)$.

Remark 3. A result of type [11, in the case $\alpha=0$, is proved in [22] using the method of Mourre commutators and micro-local analysis. In that work the potentials require only very slight polynomial decay, but they are also assumed to be infinitely differentiable, with the derivatives satisfying a symbol-like decay condition.

Clearly it would suffice to construct the operator inverse of $I+R_{0}\left(\lambda^{2}+i 0\right) L$ in a suitable weighted space $L^{2,-\sigma}$, with uniform control over its norm. In the scalar $(A=0)$ case, this becomes easy for large $\lambda$ as the norm of $R_{0}\left(\lambda^{2}+i 0\right) V$ decreases to zero.

The case of a nontrivial magnetic potential is qualitatively different, in that the norm of $R_{0}\left(\lambda^{2}+i 0\right) L$ does not decay as $\lambda \rightarrow \infty$. This follows directly from the estimate (10), which is essentially constant when the free resolvent is paired with a full derivative. What we are able to show, via more delicate analysis, is that the spectral radius of these operators diminishes to zero even though their norm does not. This allows us to work instead with the inverse of $I-(-1)^{m}\left(R_{0}\left(\lambda^{2}+i 0\right) L\right)^{m}$, which exists as a convergent power series for sufficiently large $\lambda$ and $m$.

The estimate on $\left(R_{0}\left(\lambda^{2}+i 0\right) L\right)^{m}$ is loosely based on principles of stationary phase. The region of criticality for the phase function is quite extensive, but it possesses a useful geometric structure reminiscent of Volterra operators. The remaining region can be handled by more conventional non-stationary phase methods, complicated slightly by the fact that we do not wish to assume much regularity of the potential. Full details of this argument are presented in Section 4.

\section{The basic setup}

The following result is proved in [23, Theorem 4.1]. It is based on Kato's notion of smoothing operators (see [15]). We recall that for a self-adjoint operator $H$, an operator $\Gamma$ is called $H$-smooth in Kato's sense if for any $f \in \mathcal{D}\left(H_{0}\right)$,

$$
\left\|\Gamma e^{i t H} f\right\|_{L_{t}^{2} L_{x}^{2}} \leq C_{\Gamma}(H)\|f\|_{L_{x}^{2}}
$$

or equivalently, for any $f \in L_{x}^{2}$,

$$
\sup _{\varepsilon>0}\left\|\Gamma R_{H}(\lambda \pm i \varepsilon) f\right\|_{L_{\lambda}^{2} L_{x}^{2}} \leq C_{\Gamma}(H)\|f\|_{L_{x}^{2}} .
$$


We shall call $C_{\Gamma}(H)$ the smoothing bound of $\Gamma$ relative to $H$. Let $\Omega \subset \mathbb{R}$ and let $P_{\Omega}$ be a spectral projection of $H$ associated with $\Omega$. We say that $\Gamma$ is $H$-smooth on $\Omega$ if $\Gamma P_{\Omega}$ is $H$-smooth. We denote the corresponding smoothing bound by $C_{\Gamma}(H, \Omega)$. It is not difficult to show (see e.g. [21]) that, equivalently, $\Gamma$ is $H$-smooth on $\Omega$ if

$$
\sup _{\beta>0}\left\|\chi_{\Omega}(\lambda) \Gamma R_{H}(\lambda \pm i \beta) f\right\|_{L_{\lambda}^{2} L_{x}^{2}} \leq C_{\Gamma}(H, \Omega)\|f\|_{L_{x}^{2}}
$$

The first conclusion (6) of Theorem 1 is obtained by applying Proposition 4 below. The related inhomogeneous estimate (7) then follows from (6) in a standard fashion via the Christ-Kiselev lemma [3]. The remainder of the paper will therefore be devoted to verifying the conditions needed in Proposition 4 Along the way we establish the smoothing bound (8) as an additional consequence of these conditions.

Proposition 4. Let $H_{0}=-\Delta$ and $H=H_{0}+L$ with $L=\sum_{j=1}^{J} Y_{j}^{*} Z_{j}$. Assume that each $Y_{j}$ is $H_{0}$-smooth with a smoothing bound $C_{B}\left(H_{0}\right)$ and that for some $\Omega \subset \mathbb{R}$ the operators $Z_{j}$ are $H$-smooth on $\Omega$ with a smoothing bound $C_{A}(H, \Omega)$. Assume also that the unitary semigroup $e^{i t H_{0}}$ satisfies the estimate

$$
\left\|e^{i t H_{0}} \psi_{0}\right\|_{L_{t}^{q} L_{x}^{r}} \leq C_{H_{0}}\left\|\psi_{0}\right\|_{L_{x}^{2}}
$$

for some $q \in(2, \infty]$ and $r \in[1, \infty]$. Then the semigroup $e^{i t H}$ associated with $H=$ $H_{0}+L$, restricted to the spectral set $\Omega$, also satisfies the estimate [16], i.e.,

$$
\left\|e^{i t H} P_{\Omega} \psi_{0}\right\|_{L_{t}^{q} L_{x}^{r}} \leq J C_{H_{0}} C_{B}\left(H_{0}\right) C_{A}(H, \Omega)\left\|\psi_{0}\right\|_{L_{x}^{2}}
$$

We refer the reader to [23] for the proof. Note that this approach does not capture the Keel-Tao endpoint (which would correspond to $q=2$ ) - the reason being the ChristKiselev lemma which is used in the proof of Proposition 4.

To apply this proposition we write, with a decreasing weight $w(x)=\langle x\rangle^{-\sigma}$, for some sufficiently large $\sigma>0$,

$$
\begin{aligned}
L & =2 i A \cdot \nabla+i \operatorname{div} A+V \\
& =2 i A w^{-1} \cdot \nabla\langle\nabla\rangle^{-1 / 2}\langle\nabla\rangle^{1 / 2} w+2 i A \cdot \nabla\left(w^{-1}\right) w+i \operatorname{div} A+V \\
& =\sum_{j=1}^{2} Y_{j}^{*} Z_{j}
\end{aligned}
$$

where

$$
\begin{array}{ll}
Y_{1}^{*}:=2 i A w^{-1} \cdot \nabla\langle\nabla\rangle^{-1 / 2}, & Z_{1}:=\langle\nabla\rangle^{1 / 2} w, \\
Y_{2}^{*}:=\left[2 i A \cdot \nabla\left(w^{-1}\right) w+i \operatorname{div} A+V\right] w^{-1}, & Z_{2}:=w .
\end{array}
$$

Once the operators $Z_{1}$ and $Z_{2}$ are shown to be $H$-smooth on the interval $\Omega=[0, \infty)$, the Kato smoothing estimate (8) in Theorem 1 follows immediately. 
Throughout this paper, we shall treat $\sigma>0$ as a parameter. In various places we shall specify how large it needs to be chosen. Eventually, we shall require $\sigma>4$, which will lead to the condition (4). It is standard that $Y_{1}$ and $Y_{2}$ are $H_{0}$-smooth provided

$$
|A(x)|+|\operatorname{div} A(x)|+|V(x)| \lesssim\langle x\rangle^{-1-\sigma-\varepsilon} .
$$

We now start discussing the smoothing properties of $Z_{1}$ and $Z_{2}$ relative to $H$. It will suffice to discuss $Z_{1}$.

Let us first consider intermediate energies $\lambda^{2}$, i.e., $\lambda \in\left[\lambda_{0}^{-1}, \lambda_{0}\right]=\mathcal{J}_{0}$ with $\lambda_{0}$ large. Then it was shown in [11] (see also [1]) that the resolvent of $H$ satisfies the bound

$$
\sup _{\lambda \in \mathcal{J}_{0}}\left\|\langle x\rangle^{-1 / 2-\varepsilon}\langle\nabla\rangle R_{L}\left(\lambda^{2}+i 0\right) f\right\|_{2} \leq C\left(\lambda_{0}\right)\left\|\langle x\rangle^{1 / 2+\varepsilon}\langle\nabla\rangle^{-1} f\right\|_{2}
$$

(in fact, a stronger bound was proved in [11]). More precisely, this bound follows provided there are no eigenvalues of $H$ in the interval $\mathcal{J}_{0}$. The latter property (absence of imbedded eigenvalues) is shown in [18] to hold for the entire family of potentials under consideration. An elementary proof, following [8], is also possible if one assumes that $\nabla V(x)$ exists and possesses moderate pointwise decay. It is therefore safe to conclude that

$$
\sup _{\lambda \in \mathcal{J}_{0}}\left\|Z_{1} R_{L}\left(\lambda^{2}+i 0\right) Z_{1}^{*}\right\|_{2 \rightarrow 2} \leq C\left(\lambda_{0}\right)\left\|\langle\nabla\rangle^{1 / 2} w\langle\nabla\rangle^{-1}\langle x\rangle^{1 / 2+\varepsilon}\right\|_{2 \rightarrow 2}^{2} \leq C\left(\lambda_{0}\right)
$$

since $\left\|\langle\nabla\rangle^{1 / 2} w\langle\nabla\rangle^{-1}\langle x\rangle^{1 / 2+\varepsilon}\right\|_{2 \rightarrow 2}<\infty$ by pseudo-differential calculus. Finally, by Kato's smoothing theory (see [21, Theorem XIII.30]), we conclude that $Z_{1}$ is $H$-smooth on $\Omega=\mathcal{J}_{0}$.

Note that this argument does not carry over to $\lambda \rightarrow \infty$ (in other words, for magnetic potentials, unlike the case of $V$ alone, large energies are not easy). This is due to the fact that the limiting absorption principles in [11] and [1] do not yield a gain of one derivative uniformly in $\lambda$. We devote Section 4 to this issue.

Next, we turn to small energies.

\section{Small energies}

As usual, this is reduced to zero energy. For the latter, we need to impose an invertibility condition which amounts to boundedness of the resolvent $R_{L}(0)$ between suitable spaces. More precisely, by the resolvent identity,

$$
R_{L}\left(\lambda^{2}+i 0\right)=\left(1+R_{0}\left(\lambda^{2}+i 0\right) L\right)^{-1} R_{0}\left(\lambda^{2}+i 0\right)
$$

provided the inverse on the right-hand side exists. Therefore,

$$
\begin{aligned}
\left\|Z_{1} R_{L}\left(\lambda^{2}+i 0\right) Z_{1}^{*}\right\|_{2 \rightarrow 2} & =\left\|Z_{1}\left(1+R_{0}\left(\lambda^{2}+i 0\right) L\right)^{-1} Z_{1}^{-1} Z_{1} R_{0}\left(\lambda^{2}+i 0\right) Z_{1}^{*}\right\|_{2 \rightarrow 2} \\
\leq & \left\|Z_{1}\left(1+R_{0}\left(\lambda^{2}+i 0\right) L\right)^{-1} Z_{1}^{-1}\right\|_{2 \rightarrow 2}\left\|Z_{1} R_{0}\left(\lambda^{2}+i 0\right) Z_{1}^{*}\right\|_{2 \rightarrow 2} .
\end{aligned}
$$


By the smoothing properties of $Z_{1}$ relative to $H_{0}$,

$$
\sup _{\lambda}\left\|Z_{1} R_{0}\left(\lambda^{2}+i 0\right) Z_{1}^{*}\right\|_{2 \rightarrow 2}<\infty
$$

provided $\sigma>1$. For $\lambda>1$ this follows from Agmon [1] with $\sigma>1 / 2$, whereas for small $\lambda$ it can be reduced to a Hilbert-Schmidt norm provided $\sigma>1$ (see [12]).

Thus, we need to verify that

$$
\begin{aligned}
\sup _{|\lambda|<\lambda_{0}^{-1}} \| Z_{1}(1+ & \left.R_{0}\left(\lambda^{2}+i 0\right) L\right)^{-1} Z_{1}^{-1} \|_{2 \rightarrow 2} \\
& =\sup _{|\lambda|<\lambda_{0}^{-1}}\left\|\langle\nabla\rangle^{1 / 2} w\left(1+R_{0}\left(\lambda^{2}+i 0\right) L\right)^{-1} w^{-1}\langle\nabla\rangle^{-1 / 2}\right\|_{2 \rightarrow 2}<\infty
\end{aligned}
$$

for some choice of large $\lambda_{0}$. First, we consider the case $\lambda=0$. As usual, we let $G:=$ $R_{0}(0)$.

Lemma 5. Assume that $L=2 i \nabla \cdot A-i \operatorname{div} A+V$ satisfies $|A(x)| \lesssim\langle x\rangle^{-\sigma-1-\varepsilon}$ and $|\operatorname{div} A(x)|+|V(x)| \lesssim\langle x\rangle^{-2 \sigma}$ with $\sigma>1$. Then $Z_{1} G L Z_{1}^{-1}$ is a compact operator on $L^{2}$.

Proof. First, we consider only the $2 i \nabla \cdot A$ part of $L$. We claim that

$$
\left\|\langle\nabla\rangle G \nabla \cdot A w^{-1} f\right\|_{2} \lesssim\|f\|_{2} .
$$

To see this, observe that by Plancherel

$$
\left\|D^{\alpha} G \nabla \cdot A w^{-1} f\right\|_{2} \lesssim\left\|A w^{-1} f\right\|_{2} \lesssim\|f\|_{2}
$$

provided $|\alpha|=1$. On the other hand, we will show that

$$
\left\|G \nabla \cdot A w^{-1} f\right\|_{2} \lesssim\left\|A w^{-1} f\right\|_{L^{2,1+\varepsilon}} \lesssim\|f\|_{2} .
$$

It suffices to prove that multiplication by $\xi /|\xi|^{2}$ maps $H^{1+\varepsilon}$ to $L^{2}$. Let $\chi(\xi)$ be a smooth cut-off around zero. Then $(1-\chi(\xi)) \xi /|\xi|^{2}$ maps $H^{1+\varepsilon}$ to itself, which is even stronger. Moreover, by Hölder's inequality and Sobolev imbedding,

$$
\left\|\chi(\xi)|\xi|^{-1} g\right\|_{2} \leq\left\|\chi(\xi)|\xi|^{-1}\right\|_{L^{3-}}\|g\|_{L^{6+}} \lesssim\|g\|_{H^{1+\varepsilon}},
$$

which implies 23). In conclusion, we have proved (22).

Thus,

$$
\langle\nabla\rangle^{1 / 2} w G \nabla \cdot A w^{-1}\langle\nabla\rangle^{-1 / 2}=\langle\nabla\rangle^{1 / 2} w\langle\nabla\rangle^{-1}\langle\nabla\rangle G \nabla \cdot A w^{-1}\langle\nabla\rangle^{-1 / 2}
$$

is compact in $L^{2}$, since $\langle\nabla\rangle^{1 / 2} w\langle\nabla\rangle^{-1}$ is compact in $L^{2}$.

Second, we discuss the $\widetilde{V}:=-i \operatorname{div} A+V$ part of $L$. It will suffice to show that

$$
\left\|\langle\nabla\rangle^{1 / 2} w G \tilde{V} w^{-1}\langle x\rangle^{\varepsilon} f\right\|_{2} \lesssim\|f\|_{2}
$$

since then

$$
\langle\nabla\rangle^{1 / 2} w G \tilde{V} w^{-1}\langle\nabla\rangle^{-1 / 2}=\langle\nabla\rangle^{1 / 2} w G \tilde{V} w^{-1}\langle x\rangle^{\varepsilon}\langle x\rangle^{-\varepsilon}\langle\nabla\rangle^{-1 / 2}
$$


is compact. To prove (24), we argue as before:

$$
\left\|\langle\nabla\rangle^{1 / 2} w G \tilde{V} w^{-1}\langle x\rangle^{\varepsilon} f\right\|_{2} \lesssim\left\|\nabla w G \tilde{V} w^{-1}\langle x\rangle^{\varepsilon} f\right\|_{2}+\left\|w G \tilde{V} w^{-1}\langle x\rangle^{\varepsilon} f\right\|_{2} .
$$

The second summand on the right-hand side is controlled by the Hilbert-Schmidt norm provided $\sigma>1$. The first summand is handled similarly to the proof of [23].

The following remark will be used to analyze the condition at energy zero.

Remark 6. Combining 22 with the usual boundedness properties of $G$ on weighted $L^{2}$ spaces (i.e., $G: L^{2, \beta_{1}} \rightarrow L^{2,-\beta_{2}}$ provided $\beta_{1}+\beta_{2}>2$ and $\beta_{1}, \beta_{2}>1 / 2$, see [12] or [10]) yields

$$
\|G L h\|_{L^{2,-\tau+\varepsilon / 2}\left(\mathbb{R}^{3}\right)} \leq\|h\|_{L^{2,-\tau}\left(\mathbb{R}^{3}\right)}
$$

for any $\tau>(1+\varepsilon) / 2$ provided $|\operatorname{div} A(x)|+|V(x)| \lesssim\langle x\rangle^{-2-\varepsilon}$ and $|A(x)| \lesssim\langle x\rangle^{-\tau-1-\varepsilon}$.

As an immediate consequence we arrive at the following.

Corollary 7. Assume that $\operatorname{ker}\left(I+Z_{1} G L Z_{1}^{-1}\right)=\{0\}$ as an operator on $L^{2}\left(\mathbb{R}^{3}\right)$. Then $I+Z_{1} G L Z_{1}^{-1}$ is invertible on $L^{2}$. Moreover,

$$
\left\|Z_{1}\left(I+R_{0}\left(\lambda^{2}+i 0\right) L\right)^{-1} Z_{1}^{-1}\right\|_{2 \rightarrow 2}<\infty
$$

uniformly for small $\lambda$. An analogous statement holds with $Z_{2}$ instead of $Z_{1}$.

Proof. The first statement is Fredholm's alternative. Note that

$$
\left(I+Z_{1} G L Z_{1}^{-1}\right)^{-1}=Z_{1}(I+G L)^{-1} Z_{1}^{-1}
$$

where $G L$ on the right-hand side is an operator on $Z_{1}^{-1}\left(L^{2}\left(\mathbb{R}^{3}\right)\right)$. By the same token, 26 is the same as

$$
\left\|\left(I+Z_{1} R_{0}\left(\lambda^{2}+i 0\right) L Z_{1}^{-1}\right)^{-1}\right\|_{2 \rightarrow 2}<\infty
$$

uniformly for small $\lambda$. To prove this, we write

$$
I+Z_{1} R_{0}\left(\lambda^{2}+i 0\right) L Z_{1}^{-1}=I+Z_{1} G L Z_{1}^{-1}+Z_{1} B_{\lambda} L Z_{1}^{-1}
$$

where $B_{\lambda}=R_{0}\left(\lambda^{2}+i 0\right)-G$. By a Neumann series argument, it suffices to prove that

$$
\sup _{|\lambda|<\lambda_{0}^{-1}}\left\|Z_{1} B_{\lambda} L Z_{1}^{-1}\right\|_{2 \rightarrow 2} \rightarrow 0
$$

as $\lambda_{0} \rightarrow \infty$. We have the following bounds on the kernel of $B_{\lambda}$ :

$$
\begin{aligned}
\left|B_{\lambda}(x, y)\right| & \lesssim \frac{|\lambda|^{\gamma}}{|x-y|^{1-\gamma}}, \quad 0 \leq \gamma \leq 1, \\
\left|\nabla_{x} B_{\lambda}(x, y) \nabla_{y}\right| & \lesssim \frac{\lambda}{|x-y|^{2}}+\frac{\lambda^{2}}{|x-y|}, \\
\left|\nabla_{x} B_{\lambda}(x, y)\right|+\left|B_{\lambda}(x, y) \nabla_{y}\right| & \lesssim \frac{\lambda}{|x-y|} .
\end{aligned}
$$


To prove 27, we estimate

$$
\begin{aligned}
\left\|Z_{1} B_{\lambda} L Z_{1}^{-1}\right\|_{2 \rightarrow 2} & \lesssim\left\|\nabla w B_{\lambda} L w^{-1}\right\|_{2 \rightarrow 2}+\left\|w B_{\lambda} L w^{-1}\right\|_{2 \rightarrow 2} \\
& \lesssim\left\|w \nabla B_{\lambda} L w^{-1}\right\|_{2 \rightarrow 2}+\left\|w B_{\lambda} L w^{-1}\right\|_{2 \rightarrow 2} .
\end{aligned}
$$

As before, we write $L=2 i \nabla \cdot A+\widetilde{V}$. To conclude the argument, one now uses 28) together with Schur's lemma (for the $\lambda /|x-y|^{2}$ term) as well as the Hilbert-Schmidt norm (for the others).

We now relate the condition in Corollary 7 to the notion of resonance and/or eigenvalue at zero.

Lemma 8. Suppose that zero is neither an eigenvalue nor a resonance of $H$. Then under the conditions of Lemma 5 one has

$$
\operatorname{ker}\left(I+Z_{j} G L Z_{j}^{-1}\right)=\{0\} \text { on } L^{2}\left(\mathbb{R}^{3}\right)
$$

for $j=1$, 2. In particular, 26. holds for small $\lambda$.

Proof. Suppose $f \in L^{2}\left(\mathbb{R}^{3}\right)$ satisfies

$$
f+Z_{1} G L Z_{1}^{-1} f=0 .
$$

Set $h:=Z^{-1} f$. Then $h=-G L h \in L^{2,-\sigma}\left(\mathbb{R}^{3}\right)$. Applying Remark 6 we see that $h \in$ $L^{2,-(\sigma-\varepsilon / 2)}\left(\mathbb{R}^{3}\right)$. Repeating this process shows that $h \in \bigcap_{\tau>1 / 2} L^{2,-\tau}\left(\mathbb{R}^{3}\right)$. It follows (see [12] and [10]) that $H h=0$ in the distributional sense. However, by our assumption on zero energy it follows that $h=0$ and therefore $f=0$ as desired. The argument for $Z_{2}$ is analogous.

\section{Large energies}

The goal of this section is to prove the bound

$$
\sup _{\lambda>\lambda_{0}}\left\|Z_{1} R_{L}\left(\lambda^{2}+i 0\right) Z_{1}^{*}\right\|_{2 \rightarrow 2}<\infty
$$

with some large $\lambda_{0}$ and similarly for $Z_{2}$. Here $Z_{1}, Z_{2}$ are as in (19) with $w(x)=\langle x\rangle^{-\sigma}$. Note that in combination with the previous sections this will finish the proof of Theorem[1 In order to establish (29) we introduce some notations: for any $\lambda>1$ define

$$
\widehat{T_{\lambda} f}(\xi)=\langle\xi / \lambda\rangle^{-1} \widehat{f}(\xi) \quad \text { and } \quad S_{\lambda}:=T_{\lambda}^{-1} R_{0}\left(\lambda^{2}+i 0\right) .
$$

It is clear that for any $\tau$ one has

$$
T_{\lambda}: L^{2, \tau} \rightarrow L^{2, \tau}
$$

with a bound independent of $\lambda$. Indeed, by the Fourier transform this is equivalent to

$$
\langle\xi / \lambda\rangle^{-1}: H^{\tau} \rightarrow H^{\tau}
$$


as a multiplication operator with norm independent of $\lambda$. The decay in large $|\xi|$ suggests that $T_{\lambda}$ also improves local regularity. More precisely,

$$
\left\|\langle\nabla\rangle^{\alpha} T_{\lambda} f\right\|_{L^{2 \tau}} \lesssim\langle\lambda\rangle^{\alpha}\|f\|_{L^{2, \tau}}
$$

for any $\alpha$ in $[0,1]$.

The Fourier multiplier associated to $S_{\lambda}$ is less well behaved, but we still have the following bound:

Lemma 9. With $S_{\lambda}$ as before,

$$
\left\|\langle\nabla\rangle^{\alpha} S_{\lambda} f\right\|_{L^{2,-\tau}} \lesssim \lambda^{\alpha-1}\|f\|_{L^{2, \tau}}
$$

provided $\tau>1 / 2$ and $\alpha \in[0,1]$.

Proof. By algebra of operators,

$$
\langle\nabla / \lambda\rangle^{2} R_{0}\left(\lambda^{2}+i 0\right)=2 R_{0}\left(\lambda^{2}+i 0\right)-\lambda^{-2} I .
$$

Therefore, if $\tau>1 / 2$ and $\lambda>1$, then

$$
\left\|\langle\nabla / \lambda\rangle^{2} R_{0}\left(\lambda^{2}+i 0\right) f\right\|_{L^{2,-\tau}} \leq 2\left\|R_{0}\left(\lambda^{2}+i 0\right) f\right\|_{L^{2,-\tau}}+\lambda^{-2}\|f\|_{L^{2,-\tau}} \lesssim \lambda^{-1}\|f\|_{L^{2, \tau}}
$$

by Agmon's limiting absorption principle [1]. Finally, we bound

$$
\left\|\langle\nabla\rangle^{\alpha} S_{\lambda} f\right\|_{L^{2,-\tau}} \leq\left\|\langle\nabla\rangle^{\alpha} T_{\lambda}\right\|_{L^{2,-\tau} \rightarrow L^{2,-\tau}}\left\|\langle\nabla / \lambda\rangle^{2} R_{0}\left(\lambda^{2}+i 0\right) f\right\|_{L^{2,-\tau}},
$$

which finishes the proof.

Remark 10. The resolvent estimate that we used above,

$$
\left\|R_{0}\left(\lambda^{2}+i 0\right) f\right\|_{L^{2,-\tau}} \lesssim \lambda^{-1}\|f\|_{L^{2, \tau}},
$$

follows directly from the calculations in [1], but only appears as a separately stated theorem in later works such as [12].

Next, we combine $T_{\lambda}$ and $S_{\lambda}$ with $Z_{1}$ (in what follows, we will deal with $Z_{1}$, the case of $Z_{2}$ being easier):

Lemma 11. With the previous notation,

$$
\left\|Z_{1} T_{\lambda} f\right\|_{2} \lesssim \lambda^{1 / 2}\|f\|_{L^{2,-\sigma}}, \quad\left\|S_{\lambda} Z_{1}^{*} f\right\|_{L^{2,-\sigma}} \lesssim \lambda^{-1 / 2}\|f\|_{2}
$$

for all $\lambda>1$. 
Proof. First,

$$
Z_{1} T_{\lambda}=w\langle\nabla\rangle^{1 / 2} T_{\lambda}+\left[\langle\nabla\rangle^{1 / 2}, w\right] T_{\lambda} .
$$

Now, by the same Fourier argument as above,

$$
\left\|\langle\nabla\rangle^{1 / 2} T_{\lambda} f\right\|_{L^{2,-\sigma}} \lesssim \lambda^{1 / 2}\|f\|_{L^{2,-\sigma}} .
$$

Hence, the first term on the right-hand side of (32) satisfies the desired bound. On the other hand, the commutator term in 32 can be written as

$$
\left\|\left[\langle\nabla\rangle^{1 / 2}, w\right] T_{\lambda}\right\|_{L^{2,-\sigma} \rightarrow L^{2}} \leq\left\|\left[\langle\nabla\rangle^{1 / 2}, w\right] w^{-1}\right\|_{L^{2} \rightarrow L^{2}}\left\|w T_{\lambda}\right\|_{L^{2,-\sigma} \rightarrow L^{2}} \lesssim 1
$$

uniformly in $\lambda$. Indeed, $\left[\langle\nabla\rangle^{1 / 2}, w\right] w^{-1}$ is a pseudo-differential operator of order zero and is therefore $L^{2}$ bounded, whereas

$$
\left\|w T_{\lambda}\right\|_{L^{2,-\sigma} \rightarrow L^{2}} \lesssim 1
$$

by the preceding. Next, we claim that

$$
\left\|Z_{1} S_{\lambda}^{*} f\right\|_{2} \lesssim \lambda^{-1 / 2}\|f\|_{L^{2, \sigma}}
$$

which will finish the proof by duality. To prove [33], we write

$$
Z_{1} S_{\lambda}^{*}=Z_{1} T_{\lambda} T_{\lambda}^{-2} R_{0}\left(\lambda^{2}-i 0\right) .
$$

From [31,

$$
\left\|T_{\lambda}^{-2} R_{0}\left(\lambda^{2}-i 0\right) f\right\|_{L^{2,-\sigma}} \lesssim \lambda^{-1}\|f\|_{L^{2, \sigma}}
$$

provided $\sigma>1 / 2$. Secondly, we have already shown that

$$
Z_{1} T_{\lambda}: L^{2,-\sigma} \rightarrow L^{2}
$$

with bound $\lambda^{1 / 2}$. Thus, 33 follows and we are done.

Now we continue with the proof of 29]. By the resolvent identity, we have

$$
Z_{1} R_{L}\left(\lambda^{2}+i 0\right) Z_{1}^{*}=Z_{1} T_{\lambda}\left(I+S_{\lambda} L T_{\lambda}\right)^{-1} S_{\lambda} Z_{1}^{*}
$$

provided $I+S_{\lambda} L T_{\lambda}$ is invertible as an operator on $L^{2,-\sigma}$. This invertibility will follow by means of a partial Neumann series via the following lemma. The proof of this lemma, which is the crucial technical ingredient in this paper, will be given in the next section.

Lemma 12. Given $A$ and $V$ as in Theorem 1 as well as a positive constant $c>0$, there exist sufficiently large $m=m(c)$ and $\lambda_{0}=\lambda_{0}(c)$ such that

$$
\sup _{\lambda>\lambda_{0}}\left\|\left(R_{0}\left(\lambda^{2}+i 0\right) L\right)^{m}\right\|_{L^{2,-\sigma} \rightarrow L^{2,-\sigma}} \leq c .
$$

Here $\sigma>4$.

In view of Lemma 11, the estimate in 29] follows from the following result: 
Corollary 13. With the above notation and for $\sigma>4$, we have

$$
\left(I+S_{\lambda} L T_{\lambda}\right)^{-1}: L^{2,-\sigma} \rightarrow L^{2,-\sigma}
$$

with a uniform norm for all large $\lambda$.

Proof. We write the partial Neumann series, with $m$ as in Lemma 12 .

$$
\left(I+S_{\lambda} L T_{\lambda}\right)^{-1}=\left(\sum_{k=0}^{m}(-1)^{k}\left(S_{\lambda} L T_{\lambda}\right)^{k}\right)\left(I+(-1)^{m+1}\left(S_{\lambda} L T_{\lambda}\right)^{m+1}\right)^{-1} .
$$

By Lemma 12 the inverse on the right-hand side exists on $L^{2,-\sigma}$ with a uniform bound for all $\lambda>\lambda_{0}$. Indeed,

$$
\left(S_{\lambda} L T_{\lambda}\right)^{m+1}=S_{\lambda} L\left(R_{0}\left(\lambda^{2}+i 0\right) L\right)^{m} T_{\lambda}
$$

so that, with some constant $C_{1}$ that only depends on $A$ and $V$,

$$
\begin{aligned}
\|\left(S_{\lambda} L T_{\lambda}\right)^{m+1} & \|_{L^{2,-\sigma} \rightarrow L^{2,-\sigma}} \\
& \leq\left\|S_{\lambda} L\right\|_{L^{2,-\sigma} \rightarrow L^{2,-\sigma}}\left\|\left(R_{0}\left(\lambda^{2}+i 0\right) L\right)^{m}\right\|_{L^{2,-\sigma} \rightarrow L^{2,-\sigma}}\left\|T_{\lambda}\right\|_{L^{2,-\sigma} \rightarrow L^{2,-\sigma}} \\
& \leq C_{1} c<1 / 2
\end{aligned}
$$

provided $c$ was chosen sufficiently small. Furthermore,

$$
S_{\lambda} L T_{\lambda}=2 i S_{\lambda} A \cdot \nabla T_{\lambda}+S_{\lambda}(i \operatorname{div} A+V) T_{\lambda} .
$$

By (30) and Lemma 9 .

$$
\left\|S_{\lambda}(i \operatorname{div} A+V) T_{\lambda} f\right\|_{L^{2,-\sigma}} \lesssim\|f\|_{L^{2,-\sigma}} .
$$

Furthermore, again from 30 and Lemma 9 ,

$$
\left\|S_{\lambda} A \cdot \nabla T_{\lambda}\right\|_{L^{2,-\sigma} \rightarrow L^{2,-\sigma}} \lesssim\left\|S_{\lambda} A\right\|_{L^{2,-\sigma} \rightarrow L^{2,-\sigma}}\left\|\nabla T_{\lambda}\right\|_{L^{2,-\sigma} \rightarrow L^{2,-\sigma}} \lesssim \lambda^{-1} \lambda \lesssim 1,
$$

which means the finite sum over $k=0, \ldots, m$ can be controlled with a bound independent of $\lambda$.

At this point the proof of Theorem 2 is essentially complete, thanks to the identity

$$
\begin{aligned}
\|\langle\nabla\rangle^{\alpha} R_{L} & \left(\lambda^{2}+i 0\right)\langle\nabla\rangle^{\alpha} f\left\|_{L^{2,-\sigma}}=\right\|\langle\nabla\rangle^{\alpha} T_{\lambda}\left(I+S_{\lambda} L T_{\lambda}\right)^{-1} S_{\lambda}\langle\nabla\rangle^{\alpha} f \|_{L^{2,-\sigma}} \\
& \leq\left\|\langle\nabla\rangle^{\alpha} T_{\lambda}\right\|_{L^{2,-\sigma} \rightarrow L^{2,-\sigma}}\left\|\left(I+S_{\lambda} L T_{\lambda}\right)^{-1}\right\|_{L^{2,-\sigma} \rightarrow L^{2,-\sigma}}\left\|\langle\nabla\rangle^{\alpha} S_{\lambda} f\right\|_{L^{2,-\sigma}} \\
& \lesssim\langle\lambda\rangle^{2 \alpha-1}\left\|\left(I+S_{\lambda} L T_{\lambda}\right)^{-1}\right\|_{L^{2,-\sigma} \rightarrow L^{2,-\sigma}}\|f\|_{L^{2, \sigma}} .
\end{aligned}
$$

For large $\lambda$, the desired operator bound for $\left(I+S_{\lambda} L T_{\lambda}\right)^{-1}$ is given by Corollary 13 For small $\lambda$, it follows from the Fredholm theory arguments in Section 3 One only needs to repeat the steps taken in that section using the operator $T_{\lambda}^{-1}$ in place of $Z_{1}$. 


\section{The proof of Lemma 12}

We start with the following observation: since $L=2 i \nabla \cdot A-i \operatorname{div} A+V$,

$$
\left(R_{0}\left(\lambda^{2}+i 0\right) L\right)^{m}=(2 i)^{m}\left(R_{0}\left(\lambda^{2}+i 0\right) \nabla \cdot A\right)^{m}+E_{m}\left(\lambda^{2}\right)
$$

where the error $E_{m}\left(\lambda^{2}\right)$ satisfies

$$
\left\|E_{m}\left(\lambda^{2}\right)\right\|_{L^{2,-\sigma} \rightarrow L^{2,-\sigma}} \leq C(m, V, A) \lambda^{-1}
$$

provided

$$
|A(x)|+|\operatorname{div} A(x)|+|V(x)| \lesssim\langle x\rangle^{-1-\varepsilon}
$$

This follows from Agmon's limiting absorption principle [1].

Thus, we are reduced to $L=\nabla \cdot A$. To deal with this case, we shall perform a conical decomposition of the free resolvent. Let $\left\{\chi_{\mathcal{S}}\right\}_{\mathcal{S} \in \Sigma}$ be a smooth partition of unity on the sphere $S^{2}$ which is adapted to a family of caps $\Sigma$ of diameter $\delta$ (a small parameter to be specified later). For the most part, we shall drop the subscript $\mathcal{S}$ so that $\chi$ will denote any one of these cut-offs and $\tilde{\chi}$ will typically denote a cut-off associated to $\chi$ but with a dilated cap as support. We write

$$
R_{0}\left(\lambda^{2}+i 0\right)(x)=\sum_{\mathcal{S} \in \Sigma} \frac{e^{i \lambda|x|}}{4 \pi|x|} \chi_{\mathcal{S}}(x /|x|)=: \sum_{\mathcal{S} \in \Sigma} R_{\mathcal{S}}\left(\lambda^{2}+i 0\right)(x) .
$$

We begin by studying the multiplier associated with $R_{\mathcal{S}}$.

Proposition 14. Let $\chi$ be a cut-off supported in a $\delta$-cap on $S^{2}$ where $\delta>0$ is a small parameter. Let $K_{\lambda}$ be defined as

$$
K_{\lambda}(\xi):=\mathcal{F}\left[\frac{e^{i \lambda|x|}}{4 \pi|x|} \chi(x /|x|)\right](\xi)
$$

where $\mathcal{F}$ denotes the Fourier transform. Then

$$
K_{\lambda}(\xi):= \begin{cases}O\left(\lambda^{-2} \delta^{2}\right) & \text { if }|\xi|<\lambda / 2 \\ O\left(|\xi|^{-2}\right) & \text { if }|\xi|>10 \lambda\end{cases}
$$

and for $\lambda / 2 \leq|\xi| \leq 10 \lambda$,

$$
K_{\lambda}(\xi)=O\left(\delta^{-2} \lambda^{-2}\right)+\lambda^{-1} \tilde{\chi}(\xi /|\xi|) f_{\delta}(\xi / \lambda)\left[d \sigma_{\lambda S^{2}}(\xi)+i \text { P. V. } \frac{1}{\lambda-|\xi|}\right]
$$

where $\tilde{\chi}$ is a modified cut-off supported in twice the cap of $\chi$ and $\left\|f_{\delta}\right\|_{\infty} \lesssim 1,\left\|f_{\delta}\right\|_{C^{\alpha}} \lesssim$ $\delta^{-2 \alpha}$ for any $\alpha<1$. 
Proof. By scaling, it suffices to set $\lambda=1$. Let

$$
K(\xi)=K_{\varepsilon, \delta}(\xi)=\int e^{-\varepsilon|x|} \frac{e^{i|x|}}{4 \pi|x|} \chi(x /|x|) e^{-i x \cdot \xi} d x
$$

We assume that $\chi(x)$ is smooth and supported in a $\delta$-neighborhood of $(0,0,1)$. Furthermore, by symmetry we can assume that $\xi_{2}=0$. We shall use the identity

$$
\begin{aligned}
K(\xi) & =\int_{S^{2}} \int_{0}^{\infty} e^{-\varepsilon r} e^{i r} r \chi(\omega) e^{-i r \omega \cdot \xi} d r d \sigma(\omega) \\
& =\int_{S^{2}}(\varepsilon-i(1-\omega \cdot \xi))^{-2} \chi(\omega) d \sigma(\omega) .
\end{aligned}
$$

Case 1: $\xi_{3} \leq 1 / 2$ and $|\xi| \leq 10$. Then from (38) we infer that $K(\xi)=O\left(\delta^{2}\right)$.

Case $2:\left|\xi_{3}\right| \geq|\xi| / 2$ and $|\xi|>10$. In this case $|1-\omega \cdot \xi| \gtrsim|\xi|$ so that $|K(\xi)| \lesssim \delta^{2} /|\xi|^{2}$ from (38).

Cases 3 and 4 below deal with $|\xi|>10,\left|\xi_{3}\right|<|\xi| / 2$. Note that then

$$
\{\omega \cdot \xi: \omega \in 2 \mathcal{S}\}=[a(\xi), b(\xi)]
$$

where $\mathcal{S}:=\operatorname{supp}(\chi) \subset S^{2}$ and $b(\xi)-a(\xi) \lesssim \delta$. Moreover, $2 \mathcal{S}$ denotes the twice dilated set $\mathcal{S}$.

Case 3: $\left|\xi_{3}\right| \leq|\xi| / 2$ and $|\xi|>10$, with $1 \notin[|\xi| a(\xi),|\xi| b(\xi)]$. Then

$$
\begin{aligned}
|K(\xi)| & \lesssim \int_{a(\xi)+\delta}^{b(\xi)-\delta} \frac{\delta d s}{(1-s|\xi|)^{2}} \lesssim \frac{1}{|\xi|} \int_{1-(b(\xi)-\delta)|\xi|}^{1-(a(\xi)+\delta)|\xi|} \frac{\delta}{u^{2}} d u \\
& \lesssim \frac{\delta}{|\xi|}\left(|1-(b(\xi)-\delta)| \xi||^{-1}+|1-(a(\xi)+\delta)| \xi||^{-1}\right) \lesssim \frac{\delta}{|\xi|} \frac{1}{\delta|\xi|} \lesssim|\xi|^{-2}
\end{aligned}
$$

as claimed.

Case 4: $\left|\xi_{3}\right| \leq|\xi| / 2$ and $|\xi|>10$, with $1 \in[|\xi| a(\xi),|\xi| b(\xi)]$. Here we write

$$
K(\xi)=\int_{I} \frac{\delta \psi(s)}{(s|\xi|-1-i \varepsilon)^{2}} d s
$$

where $I$ is an interval of size $\sim \delta$ centered at $|\xi|^{-1}$ and $\left|\psi^{(\ell)}(s)\right| \lesssim \delta^{-\ell}$. Shifting the center of $\psi$ to 0 and abusing notation, we obtain

$$
\begin{aligned}
K(\xi) & =\int_{-c \delta}^{c \delta} \frac{\delta \psi(s)}{(s|\xi|-i \varepsilon)^{2}} d s=\frac{\delta}{|\xi|} \int_{-c \delta}^{c \delta} \frac{\psi^{\prime}(s) d s}{s|\xi|-i \varepsilon} \\
& =\frac{\delta}{|\xi|} \int_{-c \delta}^{c \delta} \frac{\psi^{\prime}(s)-\psi^{\prime}(0)}{s|\xi|-i \varepsilon}+\frac{\delta}{|\xi|} \int_{-c \delta}^{c \delta} \frac{\psi^{\prime}(0) d s}{s|\xi|-i \varepsilon}=O\left(|\xi|^{-2}\right)
\end{aligned}
$$

using the bounds on $\psi^{\prime}$ and $\psi^{\prime \prime}$. 
Case 5: $\xi_{3} \geq 1 / 2$ and $1 / 2 \leq|\xi| \leq 10$. In this case we write

$$
K(\xi)=O\left(\delta^{-2}\right)+\int_{\delta^{-2}}^{\infty} e^{-\varepsilon r} e^{i r} r a(r \xi) d r
$$

where

$$
a(r \xi)=\int_{S^{2}} \chi(\omega) e^{-i r \omega \cdot \xi} d \sigma(\omega) .
$$

By stationary phase

$$
a(r \xi)=\frac{e^{-i r|\xi|}}{r|\xi|}\left(\chi(\xi /|\xi|)+\widetilde{\chi}(\xi /|\xi|) \frac{\delta^{-2}}{|\xi| r}\right)+O\left(\frac{\delta^{-4}}{|\xi|^{3} r^{3}}\right) .
$$

Therefore, with $e:=\xi /|\xi|$,

$$
\begin{aligned}
& K(\xi)=O\left(\delta^{-2}\right)+\frac{\chi(e)}{|\xi|} \frac{e^{[-\varepsilon+i(1-|\xi|)] \delta^{-2}}}{\varepsilon+i(1-|\xi|)}+\frac{\tilde{\chi}(e)}{|\xi|^{2} \delta^{2}} \int_{\delta^{-2}}^{\infty} \frac{e^{[-\varepsilon+i(1-|\xi|)] r}}{r} d r \\
& =O\left(\delta^{-2}\right)+\frac{1}{\varepsilon-i(1-|\xi|)}\left[\frac{\chi(e)}{|\xi|} e^{[-\varepsilon+i(1-|\xi|)] \delta^{-2}}+\frac{\tilde{\chi}(e)}{|\xi|^{2}} e^{[-\varepsilon+i(1-\mid \xi)] \delta^{-2}}\right. \\
& \left.-\frac{\tilde{\chi}(e)}{|\xi|^{2} \delta^{2}} \int_{\delta^{-2}}^{\infty} \frac{e^{[-\varepsilon+i(1-|\xi|)] r}}{r^{2}} d r\right] \\
& =: O\left(\delta^{-2}\right)+\frac{\tilde{\chi}(e)}{\varepsilon-i(1-|\xi|)} f_{\varepsilon, \delta}(\xi) .
\end{aligned}
$$

Note that, as $\varepsilon \rightarrow 0, f_{\delta}:=\lim _{\varepsilon \rightarrow 0} f_{\varepsilon, \delta}$ satisfies

$$
\left\|f_{\delta}\right\|_{\infty} \lesssim 1, \quad\left\|f_{\delta}\right\|_{C^{\alpha}} \lesssim \delta^{-2 \alpha}
$$

for any $\alpha<1$. Furthermore, in the sense of distributions,

$$
\lim _{\varepsilon \rightarrow 0} \frac{\tilde{\chi}(e)}{\varepsilon-i(1-|\xi|)}=\tilde{\chi}(e)\left[d \sigma_{S^{2}}(\xi)+i \text { P.V. } \frac{1}{1-|\xi|}\right]
$$

Here $\tilde{\chi}$ on the right-hand side is modified to absorb any needed constants.

We shall use this result to prove Proposition 16 below, which is a version of the limiting absorption principle. First, we prove a lemma about the action of the singular part in (37) on functions.

Lemma 15. Given a function $\varphi$ in $\mathbb{R}^{3}$ and $0<\alpha<1$, define

$$
[\varphi]_{\alpha}(\xi):=\sup _{|h|<1} \frac{|\varphi(\xi)-\varphi(\xi+h)|}{|h|^{\alpha}} .
$$

Then

$\mid \int_{\mathbb{R}^{3}} \varphi(\xi)\left[\sigma_{\lambda S^{2}}(d \xi)+i\right.$ P. V. $\left.\frac{d \xi}{\lambda-|\xi|} \chi_{[\lambda-1<|\xi|<\lambda+1]}\right] \mid \lesssim\|\varphi\|_{L^{1}\left(\lambda S^{2}\right)}+C_{\alpha}\left\|[\varphi]_{\alpha}\right\|_{L^{1}\left(\lambda S^{2}\right)}$ provided the right-hand side is finite. 
Proof. It suffices to consider the principal value part. Thus,

$$
\begin{aligned}
\left|\mathrm{P} . \mathrm{V} . \int_{|| \xi|-\lambda|<1} \frac{\varphi(\xi)}{|\xi|-\lambda} d \xi\right|= & \left|\mathrm{P} . \mathrm{V} . \int_{\lambda-1}^{\lambda+1} \frac{\beta^{2} \int_{S^{2}} \varphi(\beta \theta) d \sigma(\theta)}{\beta-\lambda} d \beta\right| \\
\lesssim & \int_{\lambda-1}^{\lambda+1} \frac{\beta^{2} \int_{S^{2}}|\varphi(\beta \theta)-\varphi(\lambda \theta)| d \sigma(\theta)}{|\beta-\lambda|} d \beta \\
& +\left|\mathrm{P} . \mathrm{V} \cdot \int_{\lambda-1}^{\lambda+1} \frac{\beta^{2} \int_{S^{2}} \varphi(\lambda \theta) d \sigma(\theta)}{\beta-\lambda} d \beta\right|
\end{aligned}
$$

The second term on the right-hand side in (39) is

$$
\lesssim \lambda \int_{S^{2}}|\varphi(\lambda \theta)| d \sigma(\theta) \lesssim \lambda^{-1}\|\varphi\|_{L^{1}\left(\lambda S^{2}\right)}
$$

whereas the first term is

$$
\lesssim \int_{\lambda-1}^{\lambda+1} \beta^{2}|\beta-\lambda|^{\alpha-1}[\varphi]_{\alpha}(\lambda \theta) d \sigma(\theta) d \beta \leq C_{\alpha}\left\|[\varphi]_{\alpha}\right\|_{L^{1}\left(\lambda S^{2}\right)}
$$

as claimed.

We now turn to the limiting absorption principle. Note the decay $\lambda^{-1}$ on the right-hand side which corresponds to a gain of a derivative on the left-hand side. Also, note that the constant does not depend on $\delta$ at least if $\lambda>\delta^{-2}$.

Proposition 16. Let $w=\langle x\rangle^{-\sigma}$ with $\sigma>4$. For $\lambda>\delta^{-2}$ define the kernels

$$
\begin{aligned}
& \widetilde{Q}_{\lambda}(x, y):=w(x) \frac{e^{i \lambda|x-y|}}{|x-y|} \chi\left(\frac{x-y}{|x-y|}\right) w(y), \\
& Q_{\lambda}(x, y):=w(x) \nabla_{x} \frac{e^{i \lambda|x-y|}}{|x-y|} \chi\left(\frac{x-y}{|x-y|}\right) w(y) .
\end{aligned}
$$

Then

$$
\left\|\widetilde{Q}_{\lambda}\right\|_{2 \rightarrow 2} \leq C_{0} \lambda^{-1}, \quad\left\|Q_{\lambda}\right\|_{2 \rightarrow 2} \leq C_{0}
$$

The constant $C_{0}$ does not depend on $\delta$.

Proof. It will suffice to treat $Q_{\lambda}$. We apply Schur's lemma. Thus, using the notation of Proposition 14 (and assuming that $w$ is real-valued), we obtain

$$
\begin{aligned}
\int Q_{\lambda}(x, y) \bar{f}(y) g(x) d x d y & =\int \xi K_{\lambda}(\xi) \overline{\widehat{w} * \widehat{f}}(\xi) \widehat{w} * \widehat{g}(\xi) d \xi \\
& =\iint \xi K_{\lambda}(\xi) \widehat{w}\left(\xi-\xi_{1}\right) \widehat{w}\left(\xi-\xi_{2}\right) d \xi \widehat{f}\left(\xi_{1}\right) \widehat{g}\left(\xi_{2}\right) d \xi_{1} d \xi_{2} .
\end{aligned}
$$


The theorem follows provided we can show that

$$
\sup _{\xi_{2}} \int\left|\int \xi K_{\lambda}(\xi) \widehat{w}\left(\xi_{1}-\xi\right) \widehat{w}\left(\xi-\xi_{2}\right) d \xi\right| d \xi_{1} \lesssim 1 .
$$

First, note the bounds

$$
|\widehat{w}(\xi)| \lesssim\langle\xi\rangle^{-3-\varepsilon}, \quad|\nabla \widehat{w}(\xi)| \lesssim\langle\xi\rangle^{-3-\varepsilon} .
$$

In fact, one has rapid decay here but it is not needed. Second, it follows from Proposition 14 that $K_{\lambda}:=K_{1}+K_{2}+K_{3}$ where

$$
\begin{aligned}
& K_{1}(\xi)=O\left(\delta^{-2} \lambda^{-2}\right) \chi[|\xi|<10 \lambda] \\
& K_{2}(\xi)=O\left(|\xi|^{-2}\right) \chi[|\xi|>10 \lambda] \\
& K_{3}(\xi)=\lambda^{-1} \chi(e) f_{\delta}(\xi / \lambda)\left[d \sigma_{\lambda S^{2}}(\xi)+i \mathrm{P} . \mathrm{V} \cdot \frac{1}{\lambda-|\xi|} \chi_{[\lambda-1<|\xi|<\lambda+1]}\right] .
\end{aligned}
$$

The cut-offs here are understood to be smooth. It is easy to see that $K_{1}$ and $K_{2}$ contribute $O\left(\delta^{-2} \lambda^{-1}\right)$ and $O\left(\lambda^{-1}\right)$ to 40$)$, respectively. To bound the contribution of $K_{3}$, we use Lemma 15 . Thus, define

$$
\varphi(\xi):=\xi \chi(\xi /|\xi|) f_{\delta}(\xi / \lambda) \widehat{w}\left(\xi_{1}-\xi\right) \widehat{w}\left(\xi-\xi_{2}\right) .
$$

Then

$$
\|\varphi\|_{L^{1}\left(\lambda S^{2}\right)} \lesssim \lambda \int_{\lambda S^{2}} \chi(\xi /|\xi|)\left\langle\xi_{1}-\xi\right\rangle^{-3-\varepsilon}\left\langle\xi-\xi_{2}\right\rangle^{-3-\varepsilon} d \sigma(\xi)=: J_{\lambda}\left(\xi_{1}, \xi_{2}\right)
$$

as well as

$$
\left\|[\varphi]_{\alpha}\right\|_{L^{1}\left(\lambda S^{2}\right)} \lesssim\left((\lambda \delta)^{-1}+\left(\delta^{2} \lambda\right)^{-\alpha}\right) J_{\lambda}\left(\xi_{1}, \xi_{2}\right) \lesssim J_{\lambda}\left(\xi_{1}, \xi_{2}\right)
$$

provided $\lambda>\delta^{-2}$. In view of Lemma 15 the contribution of $K_{3}$ to 40 is bounded by

$$
\sup _{\xi_{2}} \lambda^{-1} \int J_{\lambda}\left(\xi_{1}, \xi_{2}\right) d \xi_{1} \lesssim 1
$$

and the proposition follows.

Next, we study the effect of composing two resolvents which have been restricted to disjoint conical regions.

Proposition 17. Assume that $\sigma>4$ and

$$
\sum_{|\alpha| \leq 2}\left|D^{\alpha} \widehat{A}(\xi)\right| \lesssim\langle\xi\rangle^{-3-\varepsilon} \quad \forall \xi \in \mathbb{R}^{3}
$$

where $\varepsilon>0$. Let $\mathcal{S}_{1}, \mathcal{S}_{2} \subset S^{2}$ with $\operatorname{dist}\left(\mathcal{S}_{1}, \mathcal{S}_{2}\right)>5 \delta$ where dist is the distance on $S^{2}$. Let $R_{1}\left(\lambda^{2}\right)$ and $R_{2}\left(\lambda^{2}\right)$ be the free resolvents which have been restricted to conical regions corresponding to $\mathcal{S}_{1}, \mathcal{S}_{2}$, respectively. Then

$$
\left\|w R_{1}\left(\lambda^{2}\right) \nabla \cdot A R_{2}\left(\lambda^{2}\right) \nabla w\right\|_{2 \rightarrow 2} \lesssim \delta^{-2} \lambda^{-1}
$$

provided $\lambda>\delta^{-2}$. 
Proof. We use Schur's lemma as in the proof of Proposition 16 Thus, we write

$$
\begin{aligned}
\iiint g(x) w(x) \nabla_{z} R_{1}\left(\lambda^{2}\right)(x-z) A(z) \cdot \nabla_{y} R_{2}\left(\lambda^{2}\right)(z & -y) w(y) \bar{f}(y) d x d y d z \\
& =\iint \widehat{g}(\xi) U(\xi, \eta) \widehat{\widehat{f}}(\eta) d \xi d \eta
\end{aligned}
$$

where (with real-valued $w$ )

$$
U(\xi, \eta):=\int \widehat{w}\left(\xi-\xi_{1}\right) \xi_{1} \widehat{R_{1}\left(\lambda^{2}\right)}\left(\xi_{1}\right) \widehat{A}\left(\xi_{2}-\xi_{1}\right) \xi_{2} \widehat{R_{2}\left(\lambda^{2}\right)}\left(\xi_{2}\right) \widehat{w}\left(\eta-\xi_{2}\right) d \xi_{1} d \xi_{2} .
$$

We claim that

$$
\sup _{\eta} \int_{\mathbb{R}^{3}}|U(\xi, \eta)| d \xi \lesssim \delta^{-2} \lambda^{-1}
$$

By symmetry, this will imply the proposition. Next, we write as in 442 , for the Fourier transforms $K_{\lambda}^{(j)}=\widehat{R_{j}\left(\lambda^{2}\right)}$ with $j=1,2$,

$$
K_{\lambda}^{(j)}=K_{1}^{(j)}+K_{2}^{(j)}+K_{3}^{(j)}
$$

The integral on the left-hand side of (46) is bounded by

$$
\sum_{i, j=1}^{3} \int\left|\int \widehat{w}\left(\xi-\xi_{1}\right) \xi_{1} K_{i}^{(1)}\left(\xi_{1}\right) \widehat{A}\left(\xi_{2}-\xi_{1}\right) \xi_{2} K_{j}^{(2)}\left(\xi_{2}\right) \widehat{w}\left(\eta-\xi_{2}\right) d \xi_{1} d \xi_{2}\right| d \xi
$$

Of the nine different combinations here all but $i=j=3$ are easy. Indeed, if $i=1,2$ then for any $j=1,2,3$,

$$
\begin{aligned}
\int \mid \int & \widehat{w}\left(\xi-\xi_{1}\right) \xi_{1} K_{i}^{(1)}\left(\xi_{1}\right) \widehat{A}\left(\xi_{2}-\xi_{1}\right) \xi_{2} K_{j}^{(2)}\left(\xi_{2}\right) \widehat{w}\left(\eta-\xi_{2}\right) d \xi_{1} d \xi_{2} \mid d \xi \\
& \lesssim \delta^{-2} \lambda^{-1} \int\left|\widehat{w}\left(\eta-\xi_{1}\right)\right| d \xi_{1} \int\left|\int \widehat{A}\left(\xi_{2}-\xi_{1}\right) \xi_{2} K_{j}^{(2)}\left(\xi_{2}\right) \widehat{w}\left(\eta-\xi_{2}\right) d \xi_{2}\right| d \xi d \xi_{1} \\
& \lesssim \delta^{-2} \lambda^{-1}
\end{aligned}
$$

by the discussion following (40) (in particular, recall (41)). It remains to consider $i=$ $j=3$. For this we shall use Lemma 15 . Let

$$
\begin{aligned}
G_{\lambda}\left(\xi_{1}, \eta\right) & :=\int \widehat{A}\left(\xi_{2}-\xi_{1}\right) \xi_{2} K_{3}^{(2)}\left(\xi_{2}\right) \widehat{w}\left(\eta-\xi_{2}\right) d \xi_{2} \\
& =\lambda^{-1} \int \varphi\left(\xi_{2}\right)\left[\sigma_{\lambda S^{2}}\left(d \xi_{2}\right)+i \text { P. V. } \frac{d \xi_{2}}{\lambda-\left|\xi_{2}\right|} \chi_{\left[\lambda-1<\left|\xi_{2}\right|<\lambda+1\right]}\right]
\end{aligned}
$$

with

$$
\varphi\left(\xi_{2}\right):=\widehat{A}\left(\xi_{2}-\xi_{1}\right) \xi_{2} \chi_{2}\left(\xi_{2} /\left|\xi_{2}\right|\right) f_{\delta}\left(\xi_{2} / \lambda\right) \widehat{w}\left(\eta-\xi_{2}\right)
$$


Here $\chi_{2}$ is a cut-off adapted to $\mathcal{S}_{2}$. By Lemma 15 , and [43, , 44,,

$$
\left|G_{\lambda}\left(\xi_{1}, \eta\right)\right| \lesssim \int_{\lambda S^{2}} \chi_{2}\left(\xi_{2} /\left|\xi_{2}\right|\right)\left\langle\xi_{2}-\xi_{1}\right\rangle^{-3-\varepsilon}\left\langle\eta-\xi_{2}\right\rangle^{-3-\varepsilon} d \sigma\left(\xi_{2}\right) .
$$

Note that the same estimates hold if we replace $\widehat{A}$ with $\nabla \widehat{A}$. Therefore,

$$
\left|\nabla_{\xi_{1}} G_{\lambda}\left(\xi_{1}, \eta\right)\right| \lesssim \int_{\lambda S^{2}} \chi_{2}\left(\xi_{2} /\left|\xi_{2}\right|\right)\left\langle\xi_{2}-\xi_{1}\right\rangle^{-3-\varepsilon}\left\langle\eta-\xi_{2}\right\rangle^{-3-\varepsilon} d \sigma\left(\xi_{2}\right) .
$$

In view of these estimates we can apply Lemma 15 again to obtain

$$
\begin{aligned}
& \left|\int \widehat{w}\left(\xi-\xi_{1}\right) \xi_{1} K_{3}^{(1)}\left(\xi_{1}\right) G_{\lambda}\left(\xi_{1}, \eta\right) d \xi_{1}\right| \\
& \lesssim \int_{\lambda S^{2}}\left\langle\xi-\xi_{1}\right\rangle^{-3-\varepsilon} \chi_{1}\left(\xi_{1} /\left|\xi_{1}\right|\right) \int_{\lambda S^{2}} \chi_{2}\left(\xi_{2} /\left|\xi_{2}\right|\right)\left\langle\xi_{2}-\xi_{1}\right\rangle^{-3-\varepsilon}\left\langle\eta-\xi_{2}\right\rangle^{-3-\varepsilon} d \sigma\left(\xi_{2}\right) d \sigma\left(\xi_{1}\right) .
\end{aligned}
$$

Hence the contribution of $i=j=3$ to (47) is bounded by

$$
\begin{aligned}
\iint_{\lambda S^{2}} \int_{\lambda S^{2}} & \left(\left\langle\xi-\xi_{1}\right\rangle^{-3-\varepsilon} \chi_{1}\left(\xi_{1} /\left|\xi_{1}\right|\right) \chi_{2}\left(\xi_{2} /\left|\xi_{2}\right|\right)\left\langle\xi_{2}-\xi_{1}\right\rangle^{-3-\varepsilon}\left\langle\eta-\xi_{2}\right\rangle^{-3-\varepsilon}\right) \\
& \cdot d \sigma\left(\xi_{2}\right) d \sigma\left(\xi_{1}\right) d \xi \\
& \lesssim \int_{\lambda S^{2}} \int_{\lambda S^{2}} \chi_{1}\left(\xi_{1} /\left|\xi_{1}\right|\right) \chi_{2}\left(\xi_{2} /\left|\xi_{2}\right|\right)\left\langle\xi_{2}-\xi_{1}\right\rangle^{-3-\varepsilon}\left\langle\eta-\xi_{2}\right\rangle^{-3-\varepsilon} d \sigma\left(\xi_{2}\right) d \sigma\left(\xi_{1}\right) \\
& \lesssim \frac{1}{\lambda \operatorname{dist}\left(\mathcal{S}_{1}, \mathcal{S}_{2}\right)} \lesssim \lambda^{-1} \delta^{-1} .
\end{aligned}
$$

This is again smaller than $\delta^{-2} \lambda^{-1}$, as claimed.

We now write the power on the right-hand side of 35 ) as a sum of products (dropping $\lambda^{2}+i 0$ from the resolvent):

$$
\left(R_{0} \nabla \cdot A\right)^{m}=\sum_{\mathcal{S}_{1}, \ldots, \mathcal{S}_{m} \in \Sigma} R_{\mathcal{S}_{1}} \nabla \cdot A \ldots \nabla \cdot A R_{\mathcal{S}_{m}} \nabla \cdot A .
$$

There are two types of chains $\mathcal{S}_{1}, \ldots, \mathcal{S}_{m}$ in this sum:

- if $\operatorname{dist}\left(\mathcal{S}_{i}, \mathcal{S}_{i+1}\right) \leq 5 \delta$ for all $1 \leq i \leq m-1$, then we call the chain directed;

- otherwise, we call it undirected.

For the undirected chains there is the following corollary of the previous proposition.

Corollary 18. If $\left\{\mathcal{S}_{j}\right\}_{j=1}^{m}$ is undirected, then for $\sigma>4$,

$$
\left\|R_{\mathcal{S}_{1}} \nabla \cdot A \ldots \nabla \cdot A R_{\mathcal{S}_{m}} \nabla \cdot A\right\|_{L^{2,-\sigma} \rightarrow L^{2,-\sigma}} \leq C(m, A) \delta^{-2} \lambda^{-1}
$$

provided $\lambda>\delta^{-2}$. In particular, if $\lambda>\delta^{-2}$,

$$
\left\|\sum_{\substack{\mathcal{S}_{1}, \ldots, \mathcal{S}_{m} \in \Sigma \\ \text { undirected }}} R_{\mathcal{S}_{1}} \nabla \cdot A \ldots \nabla \cdot A R_{\mathcal{S}_{m}} \nabla \cdot A\right\|_{L^{2,-\sigma} \rightarrow L^{2,-\sigma}} \leq C(m, A) \delta^{-2(m+1)} \lambda^{-1} .
$$


Proof. This follows by applying Proposition 17 to one pair of resolvents where $\operatorname{dist}\left(\mathcal{S}_{i}, \mathcal{S}_{i+1}\right)>5 \delta$; for the others, use Proposition 16 More precisely, with $i$ as specified, we write

$$
A R_{\mathcal{S}_{i}} \nabla \cdot A R_{\mathcal{S}_{i+1}} \nabla \cdot A=A w^{-1} w R_{\mathcal{S}_{i}} \nabla \cdot A R_{\mathcal{S}_{i+1}} \nabla \cdot w w^{-1} A
$$

where as usual $w(x)=\langle x\rangle^{-\sigma}$. In view of $|A(x)| \lesssim\langle x\rangle^{-2 \sigma}$ and by our assumptions on $\widehat{A}$, we apply Proposition 17 to the right-hand side of (51) to conclude that

$$
\left\|w R_{\mathcal{S}_{i}} \nabla \cdot A R_{\mathcal{S}_{i+1}} \nabla \cdot w\right\|_{2 \rightarrow 2} \lesssim \delta^{-2} \lambda^{-1} .
$$

To combine this with Proposition 16 , we insert factors of $w w^{-1}$ as follows: with $\widetilde{A}:=$ $w^{-1} A w^{-1}$

$$
\begin{aligned}
\prod_{j=1}^{m}\left(R_{\mathcal{S}_{j}} \nabla A\right)= & w^{-1}\left(w R_{\mathcal{S}_{1}} \nabla w\right) \tilde{A}\left(w R_{\mathcal{S}_{2}} \nabla w\right) \tilde{A} \cdot \ldots \\
& \ldots \tilde{A}\left(w R_{\mathcal{S}_{i}} \nabla \cdot A R_{\mathcal{S}_{i+1}} \nabla \cdot w\right) \tilde{A}\left(w R_{\mathcal{S}_{i+2}} \nabla w\right) \ldots\left(w R_{\mathcal{S}_{m}} \nabla w\right) \tilde{A} w
\end{aligned}
$$

Observe that

$$
\sup _{j}\left\|w R_{\mathcal{S}_{j}} \nabla w\right\|_{2 \rightarrow 2} \leq C
$$

uniformly in $\lambda>\delta^{-2}$ as well as $\|\widetilde{A} f\|_{2} \lesssim\|f\|_{2}$. Combining this with (52) yields (49). To pass to (50) one sums over all possible choices of undirected chains of which there are no more than $(C / \delta)^{2 m}$.

Remark 19. The summation over all possible paths is quite inefficient, as it does not take advantage of any orthogonality between different operators $R_{\mathcal{S}}$. However large the constants may be, once $A, m$, and $\delta$ are fixed, the bound in 50 still approaches zero in the limit $\lambda \rightarrow \infty$.

Finally, we turn to the directed chains. For these it will be important that $\delta m \ll$ 1 to ensure that the composition of resolvents restricted to any directed chain remains outgoing. Moreover, we will need to distinguish the near and far parts of the free resolvent kernels which are defined as follows:

$$
\begin{aligned}
& Q_{\mathcal{S}}^{0}(x, y):=w(x)\left[\nabla_{y} R_{\mathcal{S}}(x-y)\right] \chi(|x-y|<\rho) w(y), \\
& Q_{\mathcal{S}}^{1}(x, y):=w(x)\left[\nabla_{y} R_{\mathcal{S}}(x-y)\right] \chi(|x-y|>\rho) w(y),
\end{aligned}
$$

where $1=\chi(|x-y|<\rho)+\chi(|x-y|>\rho)$ is a smooth partition of unity adapted to the indicated sets. The parameter $\rho$ here is a small number depending on $m$. For the near part, we have the following refinement of Proposition 16.

Proposition 20. Under the conditions of Proposition 16 one has

$$
\left\|Q_{\mathcal{S}}^{0}\right\|_{2 \rightarrow 2} \leq C_{2} \rho, \quad\left\|Q_{\mathcal{S}}^{1}\right\|_{2 \rightarrow 2} \leq C_{2}
$$

provided $\lambda>\delta^{-2} \rho^{-1}$. Here $C_{2}$ does not depend on $\delta$. 
Proof. Because of Proposition 16 it will suffice to prove the bound on $Q_{\mathcal{S}}^{0}$. In this proof, we shall write

$$
\chi_{\rho}(x-y):=\chi(|x-y|<\rho) .
$$

Observe that $\widehat{\chi}_{\rho}$ is rapidly decaying outside of a ball of size $\lesssim \rho^{-1}$. Thus, as in the proof of Proposition 16 , and with $\widetilde{K}_{\lambda}(\xi):=\xi K_{\lambda}(\xi)$,

$$
\begin{aligned}
\int Q_{\mathcal{S}}^{0}(x, y) \bar{f}(y) g(x) d x d y=\int\left[\widetilde{K}_{\lambda} * \widehat{\chi}_{\rho}\right](\xi) \widehat{\widehat{w}} * \widehat{f}(\xi) \widehat{w} * \widehat{g}(\xi) d \xi \\
=\iint\left[\widetilde{K}_{\lambda} * \widehat{\chi}_{\rho}\right](\xi) \widehat{w}\left(\xi-\xi_{1}\right) \widehat{w}\left(\xi-\xi_{2}\right) d \xi \widehat{f}\left(\xi_{1}\right) \widehat{g}\left(\xi_{2}\right) d \xi_{1} d \xi_{2}
\end{aligned}
$$

The theorem follows provided we can show that

$$
\sup _{\xi_{2}} \int\left|\int\left[\widetilde{K}_{\lambda} * \widehat{\chi}_{\rho}\right](\xi) \widehat{w}\left(\xi_{1}-\xi\right) \widehat{w}\left(\xi-\xi_{2}\right) d \xi\right| d \xi_{1} \lesssim \rho .
$$

It follows from Proposition 14 that

$$
\widetilde{K}_{\lambda}:=\widetilde{K}_{1}+\widetilde{K}_{2}+\widetilde{K}_{3}
$$

where (with smooth cut-offs)

$$
\begin{aligned}
& {\left[\widetilde{K}_{1} * \widehat{\chi}_{\rho}\right](\xi)=O\left(\delta^{-2} \lambda^{-1}\right),} \\
& {\left[\widetilde{K}_{2} * \widehat{\chi}_{\rho}\right](\xi)=O\left(\lambda^{-1}\right)} \\
& \widetilde{K}_{3} * \widehat{\chi}_{\rho}=\lambda^{-1} \widehat{\chi}_{\rho} *\left\{\chi \mathcal{S} f_{\delta}(\cdot / \lambda)\left[\lambda d \sigma_{\lambda S^{2}}(\eta)+i \mathrm{P} . \mathrm{V} \cdot \frac{\eta}{\lambda-|\eta|} \chi_{[\lambda-1<|\eta|<\lambda+1]}\right]\right\} .
\end{aligned}
$$

We also used there that $\lambda \gg \rho^{-1}$. The contributions of (54) and 55) to (53) are treated as in Proposition 16 and yield a bound of $\delta^{-2} \lambda^{-1}<\rho$ as desired. For the contribution of 56 we note that

$$
\left|\widetilde{K}_{3} * \widehat{\chi}_{\rho}\right|(\xi) \lesssim \rho
$$

Hence, the contribution of 56 to 53 is

$$
\lesssim \rho \sup _{\xi_{2}} \iint\left|\widehat{w}\left(\xi_{1}-\xi\right) \widehat{w}\left(\xi-\xi_{2}\right)\right| d \xi d \xi_{1} \lesssim \rho
$$

as desired.

Next, we write

$$
\begin{aligned}
\sum_{\substack{\mathcal{S}_{1}, \ldots, \mathcal{S}_{m} \in \Sigma \\
\text { directed }}} R_{\mathcal{S}_{1}} \nabla \cdot A \ldots \nabla & A R_{\mathcal{S}_{m}} \nabla \cdot A \\
& =\sum_{\substack{\mathcal{S}_{1}, \ldots, \mathcal{S}_{m} \in \Sigma \\
\text { directed }}} \sum_{\varepsilon_{1}, \ldots, \varepsilon_{m}=0,1} w^{-1} Q_{\mathcal{S}_{1}}^{\varepsilon_{1}} \widetilde{A} \ldots \widetilde{A} Q_{\mathcal{S}_{m}}^{\varepsilon_{m}} \widetilde{A} w .
\end{aligned}
$$


Fix a directed chain and assume without loss of generality that it is directed along the positive $x_{1}$-axis. Since $\delta m \ll 1$, one has

$$
Q_{\mathcal{S}_{j}}^{1}(x, y)=0 \quad \text { unless } \quad x_{1}-y_{1}>\rho / 2
$$

for each $1 \leq j \leq m$. Next, we decompose

$$
\widetilde{A}=\sum_{n \in \mathbb{Z}} \widetilde{A}_{n}, \quad \widetilde{A}_{n}(x):=\widetilde{A}(x) \chi_{\left[n \rho / 2<x_{1}<(n+1) \rho / 2\right]} .
$$

We start by estimating the contribution of products consisting entirely of far kernels.

Lemma 21. Suppose that $|A(x)| \leq C_{A}\langle x\rangle^{-2 \sigma-1-\varepsilon}$ with $\sigma>4$. Then, with the previous notation,

$$
\left\|Q_{\mathcal{S}_{1}}^{1} \widetilde{A} \ldots \widetilde{A} Q_{\mathcal{S}_{m}}^{1} \widetilde{A}\right\|_{2 \rightarrow 2} \leq \frac{C_{3}^{m}}{m ! \rho^{m}}
$$

provided $\lambda>\delta^{-2}+\rho^{-1}$. The constant $C_{3}$ here depends only on $A$.

Proof. By our assumptions,

$$
\left\|\widetilde{A}_{n} f\right\|_{2} \leq C_{A}(1+|n| \rho / 2)^{-1-\varepsilon}\|f\|_{2} .
$$

Moreover, since $\sup _{1 \leq j \leq m}\left\|Q_{\mathcal{S}_{j}}^{1}\right\|_{2 \rightarrow 2} \leq C_{2}$,

$$
\begin{aligned}
\left\|Q_{\mathcal{S}_{1}}^{1} \widetilde{A} \ldots \widetilde{A} Q_{\mathcal{S}_{m}}^{1} \widetilde{A}\right\|_{2 \rightarrow 2} & \leq \sum_{n_{1}>\cdots>n_{m}}\left\|Q_{\mathcal{S}_{1}}^{1} \widetilde{A}_{n_{1}} \ldots \widetilde{A}_{n_{m-1}} Q_{\mathcal{S}_{m}}^{1} \widetilde{A}_{n_{m}}\right\|_{2 \rightarrow 2} \\
& \leq C_{2}^{m} \sum_{n_{1}>\cdots>n_{m}} \prod_{j=1}^{m}\left\|\widetilde{A}_{n_{j}}\right\|_{2 \rightarrow 2} \\
& \leq C_{A}^{m} C_{2}^{m} \sum_{n_{1}>\cdots>n_{m}} \prod_{j=1}^{m}\left(1+\left|n_{j}\right| \rho / 2\right)^{-1-\varepsilon} \\
& \leq \frac{C_{A}^{m} C_{2}^{m}}{m !} \sum_{n_{1}, \ldots, n_{m} \in \mathbb{Z}} \prod_{j=1}^{m}\left(1+\left|n_{j}\right| \rho / 2\right)^{-1-\varepsilon}=\frac{C_{3}^{m}}{\rho^{m} m !}
\end{aligned}
$$

as claimed.

Next, we turn to the general case.

Lemma 22. Under the conditions of Lemma 21 .

$$
\sum_{\varepsilon_{1}, \ldots, \varepsilon_{m}=0,1}\left\|Q_{\mathcal{S}_{1}}^{\varepsilon_{1}} \widetilde{A} \ldots \widetilde{A} Q_{\mathcal{S}_{m}}^{\varepsilon_{m}} \widetilde{A}\right\|_{2 \rightarrow 2} \leq C_{5}^{m} m^{-m / 16}
$$

where $C_{5}$ only depends on A. 
Proof. Let $\mu=\sum_{j=2}^{m} \varepsilon_{j}$. Then

$$
\begin{aligned}
& \sum_{\varepsilon_{1}, \ldots, \varepsilon_{m}=0,1}\left\|Q_{\mathcal{S}_{1}}^{\varepsilon_{1}} \tilde{A} \ldots \widetilde{A} Q_{\mathcal{S}_{m}}^{\varepsilon_{m}} \widetilde{A}\right\|_{2 \rightarrow 2} \\
& \quad \leq \sum_{\varepsilon_{1}, \ldots, \varepsilon_{m}=0,1} \sum_{n_{1}}^{\left(\varepsilon_{2}\right)} \ldots \sum_{n_{m-1}}^{\left(\varepsilon_{m}\right)} \sum_{n_{m}} C_{2}^{m} \rho^{1-\varepsilon_{1}} \rho^{m-1-\mu} \prod_{j=1}^{m}\left\|\widetilde{A}_{n_{j}}\right\|_{2 \rightarrow 2} .
\end{aligned}
$$

Here, for fixed $n_{i+1}$,

$$
\sum_{n_{i}}^{\left(\varepsilon_{i+1}\right)}= \begin{cases}\sum_{n_{i}>n_{i+1}} & \text { if } \varepsilon_{i+1}=1 \\ \sum_{n_{i+1}+3 \geq n_{i} \geq n_{i+1}} & \text { if } \varepsilon_{i+1}=0\end{cases}
$$

Now

$$
\begin{aligned}
& \leq 2 \sum_{\varepsilon_{2}, \ldots, \varepsilon_{m}=0,1} \sum_{n_{1}}^{\left(\varepsilon_{2}\right)} \ldots \sum_{n_{m-1}}^{\left(\varepsilon_{m}\right)} \sum_{n_{m}}\left(C_{A} C_{2}\right)^{m} \\
& \cdot \rho^{m-1-\mu} \prod_{j=1}^{m}\left(1+\left|n_{j}\right| \rho / 2\right)^{-1-\varepsilon} \\
\leq & \left(4 C_{A} C_{2}\right)^{m} \sum_{\ell=1}^{m-1}\left(\begin{array}{c}
m-1 \\
\ell
\end{array}\right) \frac{\rho^{\ell}}{(m-\ell-1) !}\left(\frac{C}{\rho}\right)^{m-\ell-1}
\end{aligned}
$$

by counting and symmetry as in the proof of Lemma 21 Simplifying further, we conclude that

$$
\text { 59] } \leq C_{4}^{m} \rho^{-(m-1)} \sum_{\ell=1}^{m-1}\left(\begin{array}{c}
m-1 \\
\ell
\end{array}\right) \frac{\rho^{2 \ell}}{(m-\ell-1) !} .
$$

The contribution of the sum over $\ell \geq(m-1) / 2+m / 4$ to the right-hand side of $(60)$ is at most $\left(2 C_{4}\right)^{m} \rho^{m / 2}$. On the other hand, the sum over $\ell<(m-1) / 2+m / 4$ is bounded by

$$
\left(2 C_{4}\right)^{m} \frac{\rho^{-(m-1)}}{\lfloor m / 4\rfloor !}
$$

Setting $\rho:=m^{-1 / 8}$ yields the lemma.

Using [57), Lemma 22 and the observation that there are at most $\delta^{-2} C^{m}$ directed chains we conclude that

$$
\left\|\sum_{\substack{\mathcal{S}_{1}, \ldots, \mathcal{S}_{m} \in \Sigma \\ \text { directed }}} R_{\mathcal{S}_{1}} \nabla \cdot A \ldots \nabla \cdot A R_{\mathcal{S}_{m}} \nabla \cdot A\right\|_{L^{2,-\sigma} \rightarrow L^{2,-\sigma}} \leq \delta^{-2} C_{6}^{m} m^{-m / 16} .
$$

Recall that in Lemma 12 we are given an operator $L$ (quickly reduced to the case $L=$ $\nabla \cdot A)$ and a small parameter $c>0$. Based on the value of $C_{6}(A)$ from (61) we choose $m$ and $\delta=(10 \mathrm{~m})^{-1}$ large enough so that the right side of 61) is less than $c / 2$. The bound for directed chains is independent of $\lambda$. 
For the undirected chains, we apply Corollary 18 directly. With the quantities $m$ and $\delta$ already fixed, it is easy to find $\lambda_{0}$ so that the right side of (50) is less than $c / 2$ whenever $\lambda>\lambda_{0}$. This finishes the proof of Lemma 12

Acknowledgments. The authors were partially supported by the NSF, the first author by DMS0540084, the second by DMS-0600925, and the third by DMS-0617854. The third author wishes to thank Carlos Kenig for his interest and encouragement.

\section{References}

[1] Agmon, S.: Spectral properties of Schrödinger operators and scattering theory. Ann. Scuola Norm. Sup. Pisa Cl. Sci. (4) 2, 151-218 (1975) Zbl 0315.47007 MR 0397194

[2] Avron, J. E., Herbst, I. W., Simon, B.: Schrödinger operators with magnetic fields. III. Atoms in homogeneous magnetic field. Comm. Math. Phys. 79, 529-572 (1981) Zbl 0464.35086 MR 0623966

[3] Christ, M., Kiselev, A.: Maximal functions associated with filtrations. J. Funct. Anal. 179, 409-425 (2001) Zbl 0974.47025 MR 1809116

[4] Constantin, P., Saut, J.-C.: Local smoothing properties of dispersive equations. J. Amer. Math. Soc. 1, 413-439 (1988) Zbl 0667.35061 MR 0928265

[5] Constantin, P., Saut, J.-C.: Local smoothing properties of Schrödinger equations. Indiana Univ. Math. J. 38, 791-810 (1989) Zbl 0712.35022 MR 1017334

[6] Cycon, H. L., Froese, R. G., Kirsch, W., Simon, B.: Schrödinger Operators with Application to Quantum Mechanics and Global Geometry. Springer, Berlin (1987) Zbl 0619.47005 MR 0883643

[7] Erdös, L.: Recent developments in quantum mechanics with magnetic fields. In: Spectral Theory and Mathematical Physics, Proc. Sympos. Pure Math. 76, Part 1, Amer. Math. Soc., Providence, 401-428 (2007) Zbl pre05214985 MR 2310212

[8] Froese, R., Herbst, I., Hoffmann-Ostenhof, M., Hoffmann-Ostenhof, T.: On the absence of positive eigenvalues for one-body Schrödinger operators. J. Anal. Math. 41, 272-284 (1982) Zbl 0512.35062 MR 0687957

[9] Georgiev, V., Stefanov, A., Tarulli, M.: Smoothing-Strichartz estimates for the Schrödinger equation with small magnetic potential. Discrete Contin. Dynam. Systems 17, 771-786 (2007) Zbl 1125.35077

[10] Goldberg, M., Schlag, W.: Dispersive estimates for Schrödinger operators in dimensions one and three. Comm. Math. Phys. 251, 157-178 (2004) Zbl 1086.81077| MR 2096737

[11] Ionescu, A., Schlag, W.: Agmon-Kato-Kuroda theorems for a large class of perturbations., Duke Math. J. 131, 397-440 (2006) Zbl 1092.35073 MR 2219246

[12] Jensen, A., Kato, T.: Spectral properties of Schrödinger operators and time-decay of the wave functions. Duke Math. J. 46, 583-611 (1979) Zbl 0448.35080 MR 0544248

[13] Journé, J.-L., Soffer, A.; Sogge, C. D.: $L^{p} \rightarrow L^{p^{\prime}}$ estimates for time-dependent Schrödinger operators. Bull. Amer. Math. Soc. (N.S.) 23, 519-524 (1990) Zbl 0751.35011 MR 1035837

[14] Journé, J.-L., Soffer, A., Sogge, C. D.: Decay estimates for Schrödinger operators. Comm. Pure Appl. Math. 44, 573-604 (1991) Zbl 0743.35008 MR 1105875

[15] Kato, T.: Wave operators and similarity for some non-selfadjoint operators. Math. Ann. 162, 258-279 (1965/1966) Zbl 0139.31203 MR 0190801

[16] Kato, T., Yajima, K.: Some examples of smooth operators and the associated smoothing effect. Rev. Math. Phys. 1, 481-496 (1989) Zbl 0833.47005 MR 1061120 
[17] Keel, M., Tao, T.: Endpoint Strichartz estimates. Amer. J. Math. 120, 955-980 (1998) Zbl 0922.35028 MR 1646048

[18] Koch, H., Tataru, D.: Carleman estimates and absence of embedded eigenvalues. Comm. Math. Phys. 267, 419-449 (2006) Zbl pre05159628 MR 2252331

[19] Loss, M., Thaller, B.: Scattering of particles by long-range magnetic fields. Ann. Phys. 176, 159-180 (1987) Zbl 0646.35074 MR 0893482

[20] Rauch, J.: Local decay of scattering solutions to Schrödinger's equation. Comm. Math. Phys. 61, 149-168 (1978) Zbl 0381.35023 MR 0495958

[21] Reed, M., Simon, B.: Methods of Modern Mathematical Physics. IV. Analysis of Operators. Academic Press, New York (1978) Zbl 0401.47001 MR 0493419

[22] Robert, D.: Asymptotique de la phase de diffusion à haute énergie pour des perturbations du second ordre du Laplacien. Ann. Sci. École Norm. Sup. 25, 107-134 (1992) Zbl 0801.35100 MR 1169349

[23] Rodnianski, I., Schlag, W.: Time decay for solutions of Schrödinger equations with rough and time-dependent potentials. Invent. Math. 155, 451-513 (2004) Zbl 1063.35035 MR 2038194

[24] Roux, Ph., Yafaev, D.: The scattering matrix for the Schrödinger operator with a longrange electromagnetic potential. J. Math. Phys. 44, 2762-2786 (2003) Zbl 1062.81144 MR 1982788

[25] Schlag, W.: Dispersive estimates for Schrödinger operators: A survey. In: Mathematical Aspects of Nonlinear Dispersive Equations, Princeton Univ. Press, 255-285 (2007) Zbl pre05218198 MR 2333215

[26] Sjölin, P.: Regularity of solutions to Schrödinger equations. Duke Math. J. 55, 699-715 (1987) Zbl 0631.42010 MR 0904948

[27] Stefanov, A.: Strichartz estimates for the magnetic Schrödinger equation. Adv. Math. 210, 246-303 (2007) Zbl 1121.35023 MR 2298825

[28] Strichartz, R.: Restrictions of Fourier transforms to quadratic surfaces and decay of solutions of wave equations. Duke Math. J. 44, 705-714 (1977) Zbl 0372.35001 MR 0512086

[29] Vega, L.: Schrödinger equations: Pointwise convergence to the initial data. Proc. Amer. Math. Soc. 102, 874-878 (1988) Zbl 0654.42014 MR 0934859

[30] Yafaev, D. R.: Scattering matrix for magnetic potentials with Coulomb decay at infinity. Integral Equations Operator Theory 47, 217-249 (2003) Zbl 1073.47506 MR 2002666 